\title{
Transdisciplinaridade na educação à distância: um novo paradigma no ensino de Enfermagem
}

\author{
Transdisciplinarity in distance education: a new paradigm in Nursing education
}

Transdisciplinaridad en la educación a distancia: un nuevo paradigma en la educación de Enfermería

\section{Thaís Yamasaki de Campos Martins', Rita de Cássia Ribeiro", Cláudia Prado"II}

' INACI Associação de Ensino. Universidade de São Paulo, Escola de Enfermagem, Grupo de Estudos e Pesquisas de Tecnologia da Informação nos Processos de Trabalho em Enfermagem. São Paulo-SP, Brasil.

"Universidade Federal de São Paulo, Escola Paulista de Enfermagem. Universidade de São Paulo, Escola de Enfermagem, Grupo de Estudos e Pesquisas de Tecnologia da Informação nos Processos de Trabalho em Enfermagem. São Paulo-SP, Brasil.

III Universidade de São Paulo, Escola de Enfermagem, Departamento de Orientação Profissional, Grupo de Estudos e Pesquisas de Tecnologia da Informação nos Processos de Trabalho em Enfermagem. São Paulo-SP, Brasil.

Submissão: 25/03/2010 Aprovação: 27/07/2010

\section{RESUMO}

Este artigo teve como objetivo refletir sobre a necessidade da transdisciplinaridade na educação à distância, pois a mesma pressupõe um modelo novo, transição, mudança, transformação. O objetivo da educação à distância é a utilização de suporte em ambientes virtuais e interativos de aprendizagem, permitindo assim integrar múltiplas mídias de maneira organizada para elaboração de produções, interação e socialização do conhecimento. No Brasil, o ensino de Enfermagem já conta com diversas experiências na utilização da educação à distância, com resultados positivos e promissores, demonstrando boa aceitação por parte dos estudantes e melhora no processo ensino-aprendizagem.

Descritores: Enfermagem; Educação à distancia; Educação em enfermagem; Tecnologia educacional.

\section{ABSTRACT}

This article aimed to reflect on the need of applying transdisciplinarity in distance education, since it presumes a new model, transition, change, and transformation. The goal of distance education is the supportive use in virtual and interactive learning environments, allowing the integration of multiple media tools, in an organized manner, to the production, interaction and knowledge socialization elaboration. In Brazil, nursing education has several experiences in using distance education with promising results, demonstrating good acceptance on the part of students and improvements on teaching-learning process.

Key words: Nursing; Distance education; Education, nursing; Teaching; Educational technology.

\section{RESUMEN}

Este artículo tuvo como objetivo reflexionar sobre la necesidad de la transdisciplinaridad y su uso em la educación a distancia, pues la misma presupone un nuevo modelo, transición, cambio y transformación. El objetivo de la educación a distancia es la utilización de suporte en ambientes virtuales y interactivos de aprendizaje permitiendo así la integración de medias de manera organizada para la elaboración de producciones, interacción y socialización del conocimiento. Una expectativa de la ciencia frente a la frontera del conocimiento. En Brasil, la enseñanza de Enfermería ya cuenta con diversas experiencias en la utilización de la educación a distancia, con resultados positivos y promisores, demostrando una buena aceptación por los estudiantes y mejora del proceso enseñanza-aprendizaje.

Descriptores: Enfermería; Educación a distancia; Educación en enfermería; Tecnología educativa. 


\section{INTRODUÇÃO}

As novas tecnologias de informação e comunicação (TICs) vêm modificando o paradigma do ensino/aprendizagem e das relações entre o indivíduo, o trabalho e a sociedade como hoje os conhecemos ${ }^{(1-2)}$. As inovações tecnológicas alcançadas neste século foram de fundamental importância para a concretização do fenômeno da globalização. No entanto, observa-se que a Enfermagem necessita de diferentes competências para os processos de trabalho do enfermeiro, dentre eles, a capacidade de buscar novos conhecimentos, trabalhando-os, divulgando-os, nacional e internacionalmente, bem como a capacidade de produzir conhecimento e tecnologia própria, desempenhando a profissão de forma contextualizada e em equipe $^{(3)}$.

No campo da Enfermagem, a informatização surge como um alicerce para um salto na qualidade da assistência, gerência e ensino, tornando interativa e dinâmica cada fase do processo. A construção coletiva do conhecimento pode contribuir para identificar melhores formas de fazer as coisas, conscientizando os profissionais e melhorando a qualidade e a eficiência da prestação dos serviços ${ }^{(2-3)}$. Para isso, é preciso trabalhar no sentido de promover o inter-relacionamento humano e de re-humanização do uso da tecnologia incorporada ao trabalho ${ }^{(2,4)}$.

Temos, então, a Educação à Distancia (EAD) como uma ferramenta de ensino e aprendizagem extremamente adequada e desejável às demandas educacionais decorrentes das mudanças da ordem mundial atual, tendendo, doravante, a se tornar cada vez mais um elemento regular dos sistemas educativos, necessários não apenas às demandas e/ou grupos específicos, mas assumindo funções de extrema importância, especialmente, na educação da população adulta, o que inclui o ensino superior ${ }^{(2,5)}$.

Nesse sentido, o ensino à distância é a modalidade educacional de maior inferência na sociedade moderna, em permanente mudança, exige que todos nós estejamos engajados em um processo de educação permanente que perpassa ao longo da vida. A educação convencional não consegue mais atender à demanda de formação e atualização profissional no atual sistema. A evolução tecnológica busca oferecer possibilidades na área da educação. Mas será que este ensino faz mesmo diferença na construção do conhecimento? É uma evolução, como afirmam alguns, ou questão de moda? Estamos vivendo em um mundo "líquido", isto sugere a metáfora da liquidez para caracterizar o estado da sociedade de nosso tempo, cuja característica é estar em processo de contínua mudança. Ao contrário da "modernidade sólida", a "modernidade líquida", as instituições, os estilos de vida, crenças e convicções mudam antes que tenham tempo de se solidificar em costumes, hábitos e verdades "autoevidentes"(6).

Para desenvolver a educação à distância com suporte em ambientes virtuais e interativos de aprendizagem, torna-se necessária a preparação de profissionais que possam implementar e/ou utilizar plataformas de aprendizagem condizentes com as necessidades educacionais, o que implica estruturar equipes transdisciplinares constituídas por educadores, profissionais de design, programação e desenvolvimento de ambientes computacionais para EAD, com competência na criação, gerenciamento e uso desses ambientes ${ }^{(7)}$.

As TICs são o veículo para transmissão, disseminação, transformação e criação de conteúdos que podem estar ou não associados a uma tecnologia de ensino ${ }^{(8)}$; estão presentes na maioria dos campos da atividade humana, trazendo inúmeros benefícios quanto à implementação e operacionalização das principais atividades e processos realizados pelo homem. Assim que houve a disseminação do uso da internet, diversas áreas têm procurado usufruir destes benefícios, fazendo com que a informação se torne acessível ${ }^{(9)}$.

Em relação ao ensino de Enfermagem, o uso das TICs ainda é incipiente. É necessário que as Instituições de Ensino Superior adotem políticas de investimento na capacitação tecnológica docente e discente, bem como na implementação de infraestrutura para o desenvolvimento de projetos de EAD estruturados em propostas pedagógicas que viabilizem a construção de competências, habilidades e conhecimento nas áreas de tecnologia da informação e de educação utilizando novas estratégias de ensino ${ }^{(4)}$.

A sociedade atual sugere que o estudante seja alguém que busque construir seu conhecimento, alguém flexível, que saiba lidar com as necessidades de maneira criativa e que manifeste vontade de aprender, pesquisar e saber. Assim, precisamos envolver professores e estudantes neste cenário e contextualizá-lo. Para isto, é importante pensar sobre um projeto pedagógico dos cursos superiores que contemple a perspectiva interdisciplinar e as $\operatorname{TICs}^{(10)}$.

Diante do exposto, este artigo teve como objetivo refletir sobre a necessidade da transdisciplinaridade na educação à distância em enfermagem.

\section{UMA REVISÃO CONCEITUAL}

Na Enfermagem brasileira, observa-se o crescimento da produção científica na área de tecnologias com tendência em desenvolver ambientes virtuais de aprendizagem a partir de pesquisas de mestrado e doutorado, com predomínio na formação e na capacitação dos enfermeiros na área assistencial e na educação à saúde da população(2,11).

À medida que a sociedade avança em direção a um modelo altamente especializado, individualizado e competitivo na prestação de cuidado em saúde, é importante refletir sobre os modelos de prática existentes, a fim de construir novos modelos que sejam mais colaborativos, interativos, multicapacitantes e não hierárquicos, ou seja, transdisciplinares. Jantsch, Vasconcelos, e Bibeau ${ }^{(12-14)}$ fazem uma detalhada classificação evolutiva das alternativas de interação ou integração dos distintos campos disciplinares, e definem de maneira mais contextual. Para os autores, pluridisciplinaridade implica a justaposição de diferentes disciplinas científicas que, em um processo de tratamento de uma temática unificada, efetivamente desenvolveriam relações entre si. Seria, portanto, ainda um sistema de um só nível como na multidisciplinaridade, porém os objetivos são comuns, podendo existir algum grau de cooperação mútua entre as disciplinas ${ }^{(12-14)}$, 
A pluridisciplinaridade deve ser entendida como qualquer tipo de associação entre duas ou mais disciplinas, não exigindo alterações na forma e organização do ensino(15). Entretanto, o conceito de multidisciplinaridade aparece com freqüência, ou como sinônimo de pluridisciplinaridade, ou em seu lugar ${ }^{(15)}$.

Entende-se como interdisciplinaridade qualquer forma de combinação entre duas ou mais disciplinas, objetivando-se a compreensão de um objeto a partir da confluência de pontos de vista diferentes, e tendo como objetivo final a elaboração de síntese relativa ao objeto comum ${ }^{(15)}$. Uma visão interdisciplinar, unificada e convergente implica estar presente tanto no campo da teoria como da prática, seja essa prática da intervenção social, pedagógica ou de pesquisa. Deste modo, a questão da interdisciplinaridade se aguça na área das ciências humanas ${ }^{(16) .}$ Deve ser pensada como algo que se deve entender como mais do que a pluridisciplinaridade e menos do que a transdisciplinaridade ${ }^{(17)}$.

Transdisciplinaridade é o nível máximo de integração disciplinar que seria possível alcançar, um esforço deliberado para re-ligação do saber fragmentado, é o reconhecimento da interdependência de todos os aspectos da realidade, uma grande relação e cooperação entre disciplinas diversas de tal forma que estas compartilham um mesmo paradigma, um conjunto de conceitos fundamentais e/ou elementos de um mesmo método de investigação" ${ }^{\prime \prime 15,12,18) . ~}$

Está no cerne do caos criativo que a vivência transdisciplinar possibilita a construção de estratégias de enfrentamento de problemas, passando também a ver a sociedade sob um olhar integrativo e interativo, numa dimensão muito mais que inter ou multidisciplinar, ou seja. Esse novo termo, principalmente em áreas de educação e saúde, tem sido usado com freqüência cada vez maior na literatura atual.

\section{IMPLICAÇÕES PARA A ENFERMAGEM}

No Brasil, o ensino da Enfermagem já conta com diversas experiências utilizando e produzindo softwares para estudantes de Graduação, com resultados positivos e promissores, demonstrando boa aceitação por parte do público alvo e melhora no processo ensino-aprendizagem. A maioria destes softwares busca instrumentalizar os estudantes e capacitá-los a desenvolver atividades práticas, como exame físico, preparo e administração de medicamentos, processo fisiológico do parto, entre outros $^{(2,19)}$. O principal exemplo de utilização da EAD na Graduação em Enfermagem é o uso de ambientes virtuais que favoreçam o processo de aprendizagem, mas a inserção da Enfermagem na EAD ainda é incipiente, sendo necessários capacitação docente e investimento em infraestrutura ${ }^{(4)}$.

Esta modalidade de ensino apresenta grandes vantagens em relação ao ensino formal, oferecendo maior flexibilidade e agilização do processo ensino-aprendizagem por ser um sistema interativo de todos com um, um com todos e todos com todos, ou seja, transdisciplinar favorecendo o trabalho em equipe e o crescimento de todos os envolvidos.

\section{CONSIDERAÇÕES FINAIS}

A EAD pode ser inteligente ou não, aproximar ou afastar professor/estudante. Torna-se, portanto, necessário os questionamentos: que tipo de ferramentas deve ser apresentado para uma aprendizagem significativa deste novo sujeito? Usarei as ferramentas virtuais para que e por quê? Como essas ferramentas devem ser elaboradas? Com o auxílio de quais profissionais? Em relação à educação em Enfermagem, essa ferramenta deve ser voltada apenas para uma disciplina/curso ou para todos os relacionados com o assunto?

Ao mesmo tempo, a EAD precisa ser vista com uma visão transdisciplinar, ou seja, onde todos os profissionais (profissionais da área da informação e comunicação, designers e educadores) trabalhem em equipe, interagindo entre si, de forma que produzam material didático qualificado, atendendo às características do perfil dos alunos da era contemporânea, ou seja, uma geração digital, e às demandas do mundo do trabalho.

\section{REFERÊNCIAS}

1. Zem-Mascarenhas SH. Apenenf: ambiente web de apoio ao ensino de enfermagem. In: Anais do $9^{\circ}$. Congresso Brasileiro de Informática em Saúde; 2004 nov. 7-10; Ribeirão Preto (evento na internet). São Paulo: UNIFESP; 2004 Disponível em: http://telemedicina.unifesp.br/pub/ SBIS/CBIS2004/trabalhos/arquivos/247.pdf.

2. Camacho ACLF. Análise das publicações nacionais sobre educação à distância na enfermagem. Rev Bras Enferm 2009;62(4):588-93.

3. Masseto $M$, organizador. Docência na universidade. Campinas (SP): Papirus, 2003.

4. Rodrigues RCV, Peres HHC. Panorama brasileiro do ensino de enfermagem online. Rev Esc Enferm USP. 2008;42(2).

5. Belloni MP. Educação a distância. 3ed. Campinas (SP): Autores Associados, 2003.
6. Bauman Z. Modernidade líquida. São Paulo (SP): Zahar, 2001.

7. Vianney J, Torres P, Silva E. A universidade virtual no Brasil: os números do ensino superior a distância no país em 2002. In: Anais do Seminário Internacional sobre Universidades Virtuais na América Latina e Caribe; 2003 fev. 13-14; Quito, Equador.

8. Almeida MEB. Educação à distância na internet: abordagens e contribuições dos ambientes digitais de aprendizagem. Educ Pesq. 2003;29(3):327-40.

9. United Nations Educational Scientific and Cultural Organization (UNESCO). Information and communication technology in education: a curriculum for schools and programs of teacher development. Paris; 2002.

10. Santos SGF; Marques IR. Uso dos recursos de internet na Enfermagem: uma revisão. Rev Bras Enferm 2006; 59(2). 
11. AMEM BMV. Tecnologias de Informação e Comunicação: Contribuições para o Processo interdisciplinar no Ensino Superior. Revista Brasileira de Educação Médica 2006;30(3):171-180.

12. Paul P. The diferents nivels of reality between science and tradition. Bulletin interactif du CIRET (Centre International de Recherches et Estudes Transdisciplinaires). French. 2001; 17:23-27.

13. 11Jantsch $\mathrm{E}$. The interdisciplinity and the trandisciplinity of the education and inovation. In: Ocde. (The interdisciplinity). Paris.French 1972; 98-125.

14. Vasconcelos EM. Desinstitucionalização e interdisciplinaridade em saúde mental. Cadernos IPUB. Rio de Janeiro. 1997;1(7):19-42.

15. Bibeau G. Seminaire about the interdisciplinity and the application. Montreal: Université du Montreal. Department of anthropology. Canadian. 1996.
16. Zabala A. Enfoque globalizador e pensamento complexo: uma proposta para o currículo escolar. Porto Alegre (RS): Artmed, 2002.

17. Gattás MLB. Interdisciplinaridade em cursos de graduação na área da saúde da Universidade de Uberaba - Uniube [Tese]. Ribeirão Preto (SP): Escola de Enfermagem de Ribeirão Preto, Universidade de São Paulo;2005.

18. Pombo O. Interdisciplinaridade: conceito, problema e perspectiva. In: Guimarães HM, Pombo O, Levy T, organizadores. A interdisciplinaridade: reflexão e experiência. 2ed. Lisboa: Texto Editora; 1994.

19. Scurati C, Damiano D. Interdisciplinaridad y didáctica. La Corunã: Adara; 1974.

20. Almeida MEB. Educação a distância na Internet: abordagens e contribuições dos ambientes digitais de aprendizagem. Educ Pesq. 2003;29(3):327-40. 